\title{
Spectral Phonon Transport Properties of Silicon Based on Molecular Dynamics Simulations and Lattice Dynamics
}

\author{
Asegun S. Henry ${ }^{1, *}$ and Gang Chen ${ }^{2}$ \\ ${ }^{1}$ Department of Mechanical Engineering, Massachusetts Institute of Technology, 77 Massachusetts Avenue, \\ Room 7-006, Cambridge, Massachusetts 02139, USA \\ ${ }^{2}$ Department of Mechanical Engineering, Massachusetts Institute of Technology, 77 Massachusetts Avenue, \\ Room 3-260, Cambridge, Massachusetts 02139, USA
}

\begin{abstract}
Although the thermal conductivity of silicon has been studied before, current estimations for the phonon mean free paths have not provided full explanation of the strong size effects experimentally observed for various silicon micro and nanostructures. Since phonon relaxation time models are mostly semi-empirical, the mean free paths cannot be determined reliably and questions remain as to which polarizations, frequencies and wavelengths are dominant heat carriers. Here we have used a combination of equilibrium molecular dynamics simulations and lattice dynamics calculations to fully detail the spectral dependence of phonon transport properties in bulk silicon. By considering the frequency dependence of the specific heat, group velocities and mean free paths, we address these unresolved questions and examine the errors associated with isotropic and frequency averaged approximations. Simulation details, such as the convergence of results on the simulation time and extraction of phonon transport properties in different crystallographic directions, are also discussed.
\end{abstract}

Keywords: Silicon, Molecular Dynamics, Phonons, Relaxation Time, Mean Free Path, Thermal Conductivity.

\section{INTRODUCTION}

Phonons are quantized lattice vibrations that carry energy $h \nu$ and quasi momentum $h \mathbf{k} / 2 \pi$, where $h$ is Planck's constant $\nu$ is the phonon frequency and $\mathbf{k}$ its wave vector. ${ }^{1-8}$ Phonons interact with each other, as well as boundaries, impurities and other crystal imperfections through scattering events. Peierls ${ }^{1}$ showed that these scattering events, which are subject to energy and momentum conservation constraints, can be classified as either normal processes, which conserve quasi-momentum, or umklapp process which do not. Peierls work showed that umklapp processes generate thermal resistance (finite thermal conductivity) and are necessary for the crystal to obtain thermal equilibrium. In most insulators and semiconductors, where the conduction electron density is low, phonons are the dominant energy carriers and quantitative understanding of their interactions has become increasingly important for micro/nanoscale applications. Phonon thermal conductivity, which we derive in the following section, depends

*Author to whom correspondence should be addressed. on three distinct phonon transport properties- the specific heat, group velocity and mean free path (MFP). The specific heat for each mode and group velocities $\mathbf{v}$ can be obtained from lattice dynamics, but the MFPs $\Lambda$, which are related to the relaxation times $\Lambda=\mathbf{v} \cdot \tau$, have eluded direct calculation for many years. ${ }^{1-8}$

For many nanoscale applications, the length scale reduction only has significant impact on the specific heat and group velocities at cryogenic temperatures. ${ }^{9}$ The MFPs, however, are greatly impacted by size effects over the entire temperature range. Experiments have shown that these size effects are highly important in both the micro and nanoscale regimes, where thermal conductivity can decrease by several orders of magnitude. ${ }^{10}$ Other scattering effects at these length scales can also cause heat conduction to deviate significantly from Fourier's Law. ${ }^{11}$ As a result, emerging nanotechnological applications have increased the demand for reliable quantitative results for phonon MFPs in order to accurately understand these effects-particularly for silicon, a highly important semiconductor material that is heavily used in semiconductor industries. ${ }^{12}$ Size effects are crucial for microelectronic 
components, where thermal conductivity reduction leads to increased resistance to heat dissipation and presents a serious obstacle to continued miniaturization. ${ }^{12}$ Size effects are also especially important in thermoelectric applications, where the thermal conductivity reduction is used to enhance the efficiency of nanostructured materials. ${ }^{13,14}$ For many technological applications involving bulk silicon ( $T>100 \mathrm{~K})$, phonon-phonon scattering is the dominant mechanism governing thermal conductivity. As the characteristic device length decreases, thermal conductivity decreases because boundary scattering increases and limits the MFPs. The intermediate regime where both phononphonon scattering and boundary scattering are important spans three orders of magnitude $\left(10^{-9} \mathrm{~m}-10^{-6} \mathrm{~m}\right)$. To explain size effects in this regime, estimations of an effective phonon MFP due to phonon-phonon scattering have been used to gain insight on the length scale where boundary scattering becomes dominant. A standard kinetic theory based approach, that uses the speed of sound and bulk specific heat of silicon, yields a room temperature MFP of $41 \mathrm{~nm} .{ }^{15}$ It has been recognized, however, that the average phonon velocity is much smaller than the speed of sound and that optical phonons contribute much more to the specific heat than to the thermal conductivity. Ju and Goodson ${ }^{16}$ studied the thermal conductivity of various silicon thin films, and based on their modeling, estimated an effective phonon MFP between 200-300 nm. These estimations, which differ by almost an order of magnitude, can be used in approximate models for the thermal conductivity, but they provide insufficient explanation for the strong size effects observed in silicon microstructures. ${ }^{17,18}$

A body of theoretical work qualitatively explains phonon thermal conductivity, ${ }^{1-8}$ yet it is difficult to understand and predict the strength of size effects because quantitative results for phonon MFPs are lacking. The major challenge is determining the relaxation times. Klemens ${ }^{2}$ developed an approach to calculate relaxation times using a quantum scattering matrix and Fermi's golden rule. By assuming linear isotropic phonon dispersion, Klemens estimated $\tau \propto \nu^{-2} \cdot T^{-1}$ at high temperatures. Callaway ${ }^{3}$ developed a model that accounts for both normal and umklapp processes, but neither Callaway nor Klemens' models accurately capture the thermal conductivity's temperature dependence at higher temperatures. Although Holland ${ }^{4}$ obtained better agreement by including phonon dispersion and fitting to thermal conductivity data for semiconductors, all of the semi-empirical models require multiple fitting parameters that cannot be determined reliably. This relative uncertainty in the relaxation times for different polarizations has lead to lingering questions surrounding the dominant polarization. Hamilton and Parrott $^{19}$ addressed this issue using an alternative procedure to the relaxation time approximation and solved the Boltzmann equation by applying the variational principal. By assuming a trial function and linear isotropic dispersion, Hamilton and Parrott studied the thermal conductivity of Germanium and showed that transverse acoustic (TA) phonons are responsible for $80-90 \%$ of the thermal conductivity, while longitudinal acoustic (LA) phonons contribute less than $20 \%$. Based on their work ${ }^{18}$ it has been widely accepted that TA phonons dominate. Savvides and Goldsmid $^{18}$ used Hamilton and Parrott's conclusion that TA phonons dominate to explain their experimental results with a model that assumed TA phonons as the only heat carriers. Ju and Goodson, ${ }^{16}$ on the other hand, have done more recent experiments and modeling of silicon thin films and provide the best explanation of their results by assuming LA phonons are the only heat carriers. The conflicting conclusions of these and other works have rendered the issue of identifying the dominant polarization unresolved.

Srivastava $^{20}$ showed that the off diagonal terms in threephonon collision operator play an important role at higher temperatures, above the regime where boundary scattering dominates. Srivastava also assumed linear dispersion in his work, but could not match the thermal conductivity trend at higher temperatures where $\kappa \propto T^{-n}, n>1$. This issue was also encountered by Klemens, ${ }^{2}$ Callaway $^{3}$ and Holland. ${ }^{4}$ Srivastava $^{20}$ attributed the discrepancy to the assumption of linear dispersion, temperature dependent gruneissen parameter and possibly four-phonon or higher order processes. More recently, however, Omini and Sparavigna ${ }^{21}$ have developed the closest technique to a first principle thermal conductivity calculation, which solves the BTE through an iterative procedure. Their work employed only a few minor assumptions and served to clarify the role of optical phonons as well as the importance of each scattering mechanism. The common thread between Omini and Sparavigna ${ }^{21}$ and the present work, is that the effects of dispersion, interactions with optical phonons and temperature dependent anharmonicity are all included. The various approaches chosen by different authors have generated little conclusive agreement concerning the role of different polarizations and which frequencies are most important. As a result, analytical study of phonon scattering has lead to unresolved questions concerning the details of phonon transport, particularly in silicon. Here we used an approach to calculate relaxation times without fitting parameters and show that when nonlinear dispersion and temperature dependent anharmonicity are included in the modeling, the simpler relaxation time approach leads to the same conclusions as Omini and Sparavigna's first principals calculation. ${ }^{21}$ By adopting the relaxation time approach, however, we are able to explore the contributions of different phonon frequencies, wavelengths and polarizations in greater detail to provide an explanation of the size effects observed in silicon microstructures.

In the following sections we discuss numerical simulation alternatives to analytical study of thermal conductivity and phonon transport properties. We first discuss molecular dynamics (MD) simulations and the widely used 
Green-Kubo approach to calculating thermal conductivity. We then derive an expression for thermal conductivity in terms of frequency and polarization dependent phonon transport properties, which can be used to understand size effects through the spectral dependent contributions. The remaining sections detail the simulation analysis and procedures, concluding with a discussion of various results from our simulations of bulk silicon. The numerical approach used to calculate relaxation times here does not distinguish between the effects of normal, umklapp, threephonon, four-phonon or even higher order scattering. In this modeling approach, these various effects are combined into a single relaxation time to represent the net effect of phonon-phonon scattering, but with full order anharmonic contributions.

\section{GREEN-KUBO ANALYSIS OF MOLECULAR DYNAMICS SIMULATIONS}

Analytical study of phonon scattering has confronted difficult obstacles and numerical simulation has become an increasingly popular alternative. One approach being used to study the intrinsic characteristics of materials is molecular dynamics (MD) simulation, which treats atoms as point particles and tracks their individual motions with time. Classical MD simulations use empirical expressions to model the interactions between atoms, which are typically fit to data from quantum electronic structure calculations and experiments. Several authors have used classical MD simulations to calculate the thermal conductivity of existing materials, ${ }^{22,23}$ including silicon. ${ }^{24-26}$ The most common approach uses the Green-Kubo formula, ${ }^{27}$ which expresses the thermal conductivity tensor $\kappa_{\alpha, \beta}$ in terms of its internal fluctuations, based on linear response theory

$$
\kappa_{\alpha, \beta}=\frac{V}{k_{\mathrm{B}} T^{2}} \int\left\langle\mathbf{Q}_{\alpha}\left(t+t^{\prime}\right) \cdot \mathbf{Q}_{\beta}(t)\right\rangle d t^{\prime}
$$

Expressions for the heat flux $\mathbf{Q}$ in terms of the quantities available in atomistic simulations have been derived in several ways. The derivations start from energy conservation, $(d E / d t)+\nabla \cdot \mathbf{Q}=0$, but differ in how the heat flux divergence is treated. Some derivations ${ }^{23-26}$ write the heat flux as

$$
\mathbf{Q}=\frac{d}{d t}(E \cdot \mathbf{r})
$$

Authors following this approach ${ }^{23-26}$ have used expressions such as

$$
\mathbf{Q}=\frac{1}{V} \sum_{i}\left[E_{i} \cdot \mathbf{v}_{i}+\sum_{j}\left(F_{i j} \cdot \mathbf{v}_{i}\right) \mathbf{r}_{i j}\right]
$$

to determine the heat flux in a MD simulation. The second term of Eq. (3) has variable interpretations for many-body potentials, because it is written in terms of pair-wise force interactions. Schelling et al. ${ }^{25}$ tested some of these interpretations on the Stillinger-Weber potential for silicon, but observed minimal impact on the resultant thermal conductivity. Hardy ${ }^{28}$ however, has derived a heat flux operator that uses a spatial weighting function to describe the local energy density as a continuous function. Hardy's result is valid for any system where the energy is expressed in the form

$$
E=\sum_{i} \frac{1}{2} m_{i} \mathbf{v}_{i}^{2}+\Phi_{i}
$$

where $\Phi_{i}$ is the potential energy associated with a single atom. Hardy's result ${ }^{25}$ for the heat flux operator

$$
\mathbf{Q}=\frac{1}{V} \sum_{i}\left[E_{i} \cdot \mathbf{v}_{i}+\sum_{j}\left(-\nabla_{\mathbf{r}_{i}} \Phi_{j} \cdot \mathbf{v}_{i}\right) \cdot \mathbf{r}_{i j}\right]
$$

also has two terms, but the interaction term subtly differs from Eq. (3). Hardy's result is general and can be applied to any empirical potential, provided the energy can be written in terms of individual atoms.

Equation (1) is a general result, valid for any phase of matter, but has been mostly used to calculate the thermal conductivity of liquids and solids. Since Eq. (1) describes $\kappa$ in terms of any system's response to a perturbation, the connection to phonon transport properties, such as the relaxation time is unclear. To establish this connection we seek an alternative framework that directly relates the atomic trajectory to the phonon properties. The relaxation times are calculated using the lattice wave description of phonons, where the lattice wave attenuation corresponds to phonon scattering and is studied by projecting the atomic trajectory onto the system's normal modes. This transformation to normal mode coordinates generates time dependent amplitudes, ${ }^{8}$ which are then used to extract relaxation times for individual modes and can be expanded to a full spectrum using interpolation and extrapolation. To use this information to calculate thermal conductivity, we first derive the thermal conductivity of a solid in terms of frequency dependent phonon transport properties we can determine from MD and lattice dynamics simulations.

\section{PHONON THERMAL CONDUCTIVITY}

Phonon scattering events create and annihilate phonons under the constraints of energy and momentum conservation. ${ }^{1-8}$ At equilibrium, these scattering events result in an average occupation number for each phonon state described by Bose-Einstein statistics

$$
f_{0}=\frac{1}{\exp \left(h \nu / k_{\mathrm{B}} T\right)-1}
$$

where $k_{\mathrm{B}}$ is Boltzmann's constant. When the system is in nonequilibrium, there is a net energy transport and the nonequilibrium occupation can be described by a solution to the Boltzmann transport equation (BTE). A typical approximation used to solve the BTE for phonons is the single mode relaxation time approximation (SMRT), 
which assumes that the occupation decays exponentially back to its equilibrium value through a single time constant for each mode. By assuming that the time and spatial derivatives of the distribution's deviation from equilibrium $f-f_{0}$ are negligible compared to the spatial variation of $f_{0}$, we can solve the BTE for the nonequilibrium distribution

$$
f(\mathbf{r}, \mathbf{k}, p)=f_{0}-\frac{d f_{0}}{d T} \cdot \nabla T \cdot \mathbf{v} \cdot \tau
$$

where $\mathbf{r}$ is the location within the system, the phonon state is described by wave vector $\mathbf{k}$, polarization $p$, has relaxation time $\tau$ and travels with velocity $\mathbf{v}$. With this distribution we can write the net energy flux (heat flux) carried by phonons in the $x$ direction, by summing the contributions of all phonon states with occupation $f$.

$$
J_{x}(x)=\frac{1}{V} \sum_{p} \sum_{k_{x}=-\infty}^{\infty} \sum_{k_{y}=-\infty}^{\infty} \sum_{k_{z}=-\infty}^{\infty} h \nu \cdot f \cdot \mathbf{v} \cdot \hat{\mathbf{x}}
$$

Taking the system to be large, we can convert the wave vector summations to spherical integrals over $\mathbf{k}$-space using a discrete $\mathbf{k}$-space interval of $\Delta\|\mathbf{k}\|=2 \pi / L$, where $L$ is the system's length in each direction.

$$
J_{x}(x)=\sum_{p} \int_{0}^{\infty} \int_{0}^{2 \pi} \int_{0}^{\pi} h \nu \cdot f \cdot v_{x} \cdot \frac{k^{2} d k \sin (\theta) d \theta d \varphi}{V \cdot(2 \pi / L)^{3}}
$$

Taking the $\mathbf{k}$-space as isotropic, we substitute the density of states $D(\nu)$ (the number of states within a differential frequency interval), where $(2 \pi / L)^{3}$ is the $\mathbf{k}$-space volume occupied by each state, and write Eq. (9) as two spatial integrals and an integral over the phonon frequencies.

$$
J_{x}(x)=\sum_{p} \int_{0}^{\nu_{\max }} \int_{0}^{2 \pi} \int_{0}^{\pi} h \nu \cdot f \cdot v_{x} \cdot D(\nu) \cdot \sin (\theta) d \theta d \varphi d \nu
$$

By substituting Eq. (7) for the nonequilibrium occupation and assuming isotropic velocities and relaxation times, we can carry out the spatial integrals, resulting in

$J_{x}(x)=-\left\{\sum_{p} \frac{1}{3} \int_{0}^{\nu_{\max }} \int_{0}^{2 \pi} \int_{0}^{\pi}\left[h \nu \cdot D(\nu) \cdot \frac{d f_{0}}{d T}\right] v^{2} \tau \cdot d \nu\right\} \cdot \frac{d T}{d x}$

which has the same form as Fourier's law of heat conduction, where

$$
C(\nu)=\frac{d E}{d T}=\left[h \nu \cdot D(\nu) \cdot \frac{d f_{0}}{d T}\right]
$$

is the frequency dependent specific heat per unit volume. This leads to the following expressions for thermal conductivity,

$$
\kappa=\sum_{p} \frac{1}{3} \int_{0}^{\nu_{\max }} C \cdot v^{2} \cdot \tau \cdot d \nu=\sum_{p} \frac{1}{3} \int_{0}^{\nu_{\max }} C \cdot v \cdot \Lambda \cdot d \nu
$$

where $\Lambda$ is the phonon MFP. Equation (13) allows us to investigate the spectral dependence of the phonon thermal conductivity of solids. The summation over polarizations also allows us to determine the separate contributions from each branch and identify which polarizations dominate in a particular material. In the following sections we discuss the numerical techniques used to calculate the frequency dependent phonon transport properties, using a combination of lattice dynamics and MD simulations.

\section{SIMULATION ANALYSIS}

\subsection{Frequency Dependent Specific Heat}

Equation (12) expresses the frequency dependent specific heat, where the density of states is the only unknown. Using lattice dynamics, we can solve the equations of motion under the harmonic approximation and use a Taylor expansion of the potential energy to determine the dynamical matrix. Lattice dynamics determines the phonon frequencies at any wave vector by solving for the eigen values and eigen vectors of the dynamical matrix. This is then used to calculate $D(\nu)$, by summing the number of states in each direction. By discretizing a sub-region of the Brillouin zone, outlined by the principal symmetry directions, we can directly count the number of phonon states (dynamical matrix eigen values) that fall within a particular frequency interval. This provides a direct measure of the frequency dependent density of states, which can then be normalized for each polarization, summing to three (3D) states per atom. If the counting procedure is done using small frequency intervals, we can use the discretized spectrum to calculate all the necessary phonon properties numerically, which enables us to treat the integrations as discrete summations with negligible error. A discrete representation for frequency dependent phonon properties has several advantages over analytical formulation. It accurately captures nonlinear features in the data, particularly for the relaxation times, it simplifies the process of integration and enables greater flexibility in reorganizing the data for analysis. Lattice dynamics serves two purposes in this analysis approach. First the eigen values are used in determining the density of states and second the eigen vectors are needed to transform the MD trajectory to normal mode coordinates. For this reason the lattice dynamics calculations are prerequisite to the decomposition of the MD trajectory into normal mode amplitudes, which will be discussed in detail later.

\subsection{Frequency Dependent Group Velocity}

The frequency dependent group velocity can be determined from the lattice dynamics dispersion or can be well approximated by the dispersion extracted from the MD simulations, if enough modes are analyzed. The MD dispersion may be preferred if the temperature dependence 
of the group velocities is of interest, however the lattice dynamics dispersion can be used with minimal error for many applications. The group velocities can be numerically tabulated with respect to frequency and separately stored for each polarization in different directions to allow for detailed analysis of the specific contributions to thermal conductivity.

\subsection{Frequency Dependent Relaxation Time}

The major advancement ${ }^{22,23}$ highlighted by this work is the use of the MD trajectories to determine phonon relaxation times in silicon crystals. The atomic trajectories generated by MD simulations enable direct calculation of normal mode decay times. By transforming the trajectory to normal mode coordinates, we obtain a time history of the normal mode amplitudes $A(\mathbf{k}, p, t)$.

$$
A(\mathbf{k}, p, t)=\sum_{j}\left(\mathbf{r}_{j}(t)-\mathbf{r}_{j 0}\right) \cdot \mathbf{p}_{j}(\mathbf{k}, p) \cdot \exp \left(i \cdot \mathbf{k} \cdot \mathbf{r}_{j 0}\right)
$$

The summation is carried out over the atoms $j$ within the system of interest, $\mathbf{r}_{j}(t)$ is the atom's position, $\mathbf{r}_{j 0}$ its lattice position and $\mathbf{p}_{j}(\mathbf{k}, p)$ its corresponding polarization vector (dynamical matrix eigen-vector) obtained from lattice dynamics. Equation (14) gives the plane

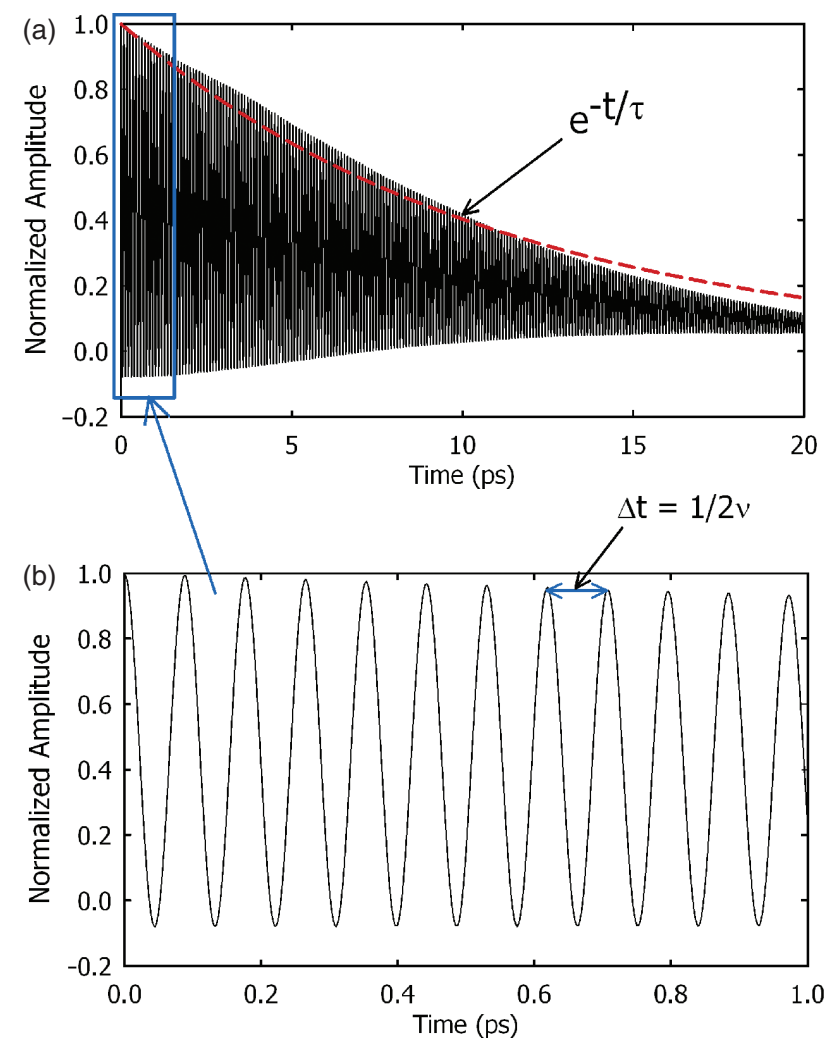

Fig. 1. Normal mode autocorrelation function. (a) Normal mode decay and exponential fit. (b) Closer view of the normal mode autocorrelation oscillations. The autocorrelation oscillation frequency is double the phonon frequency because the amplitude is squared. wave amplitude for an individual mode. ${ }^{8}$ These time varying amplitudes correspond to temporal variations in the phonon occupation and the average mode energy is proportional to the average occupation at equilibrium. It is the temporal deviations from the average, however, that characterize the interactions amongst the various phonons within the system. The normal mode autocorrelation function describes the temporal amplitude attenuation and the decay time constant is the relaxation time ${ }^{5}$ for the corresponding phonon, written as ${ }^{22}$

$$
\tau(\mathbf{k}, p)=\frac{\int\left\langle\delta A\left(\mathbf{k}, p, t+t^{\prime}\right) \cdot \delta A(\mathbf{k}, p, t)\right\rangle d t^{\prime}}{\left\langle\delta A(\mathbf{k}, p, t)^{2}\right\rangle}
$$

where $\delta A(\mathbf{k}, p, t)$ is the deviation from the average normal mode amplitude. Normal mode autocorrelation functions, based on Eq. (14), decay with oscillations because they only consider the mode's potential energy. Figure 1 shows an example normal mode autocorrelation function with the corresponding decay time and phonon frequency indicated. The temperature dependence of phonon frequencies can be extracted in this manner, as frequencies typically decrease with increasing temperature due to softening of the interactions. McGaughey and Kaviany ${ }^{23}$ showed that smoothly decaying autocorrelations are obtained by considering the mode's total energy as

$$
\begin{aligned}
E(\mathbf{k}, p, t)= & \left(\frac{A(\mathbf{k}, p, t) \cdot A^{*}(\mathbf{k}, p, t) \cdot \omega^{2}}{2}\right) \\
& +\left(\frac{\dot{A}(\mathbf{k}, p, t) \cdot \dot{A}^{*}(\mathbf{k}, p, t)}{2}\right)
\end{aligned}
$$

where $\omega$ is the angular frequency obtained from lattice dynamics and $*$ denotes the complex conjugate. Negligible error, however, is introduced by fitting the peaks of the autocorrelation function (based on Eq. (14)) with an exponential. McGaughey and Kaviany also used a factor of two in their relaxation time calculations. We apply Eq. (15) directly, similarly to Ladd et al., ${ }^{22}$ taking the time constant $\tau$ in Eq. (15) to be equal to the phonon relaxation time.

It is important to note that the relaxation times extracted from MD simulations combine the effects of three, four and higher order phonon scattering into a single relaxation time. This is due to the comprehensive inclusion of temperature dependent anharmonicity expressed through the atomic trajectory, which is driven by a nonlinear interatomic potential. As a result, this method of calculating relaxation times, does not distinguish between normal, umklapp or which polarizations are interacting, but provides a measure of the net phonon-phonon scattering rate. Using the analysis tools described in the preceding sections, we conducted various simulations of bulk silicon to study the temperature dependence of its phonon transport properties. 


\section{SIMULATION PROCEDURES}

We conducted microcanonical MD simulations using periodic boundary conditions to replicate the effects of an infinite medium. A large body of work has been invested to develop empirical potentials for silicon, with varying successes. For this study we chose the environment dependent interatomic potential (EDIP) ${ }^{29}$ because of its innovative functional form and explicit dependence on coordination. EDIP was designed to reproduce properties of the bulk phases of silicon, with strong emphasis on the elastic constants and vacancy energies. To test whether EDIP accurately captured the phenomena of interest, preliminary simulations were conducted using the Green-Kubo method. All simulations used a timestep of 1 femtosecond and a lattice constant of $0.54309 \mathrm{~nm}$. The preliminary simulations indicated that a 1 femtosecond timestep provided sufficient resolution to capture the highest frequency oscillations and also showed that thermal expansion effects generated minimal impact on the results. Figure 2 shows how the thermal conductivity calculated with the Green-Kubo method converged with longer simulation time. Figure 2(a) shows that the amount of equilibration time before the heat flux is sampled has insignificant impact on the results for long simulation times. Sun and Murthy ${ }^{26}$ tested the convergence of thermal conductivity using EDIP with increasing number of atoms at fixed simulation duration. Figure 2(b) shows that even larger simulation sizes may require long times for convergence of the heat flux autocorrelation function. We expected that shorter simulation times would be required for larger systems, yet the results do not clearly indicate faster convergence. Figure 2(c) does, however, show that the convergence time tends to decrease slightly with increasing temperature. Based on the results in Figure 2, Green-Kubo simulations were run for ten nanoseconds while similar testing was done for relaxation time simulations. As a result relaxation time simulations were run for five nanoseconds, and all simulations allowed 100 picoseconds of equilibration time.

For the results corresponding to different symmetry directions, it was observed that only specific wave vectors, which repeated in accordance with the periodic boundary conditions, generated consistent normal mode autocorrelations. Long wavelength modes and other non-eigen modes that do not share the same periodicity as the MD periodic boundaries primarily oscillate at several frequencies as opposed to the single frequency of the corresponding mode. As a result the non-eigen mode autocorrelations decayed more rapidly and behaved inconsistently. For cubic domains this introduced constraints on the number of modes that could be analyzed. To overcome the constraint, modified simulation cells, shown in Figure 3, were used to reorient the direction of interest with the longest edge of a rectangular domain. This proved advantageous over cubic domains where fewer eigen modes are available for analysis in diagonally oriented directions.
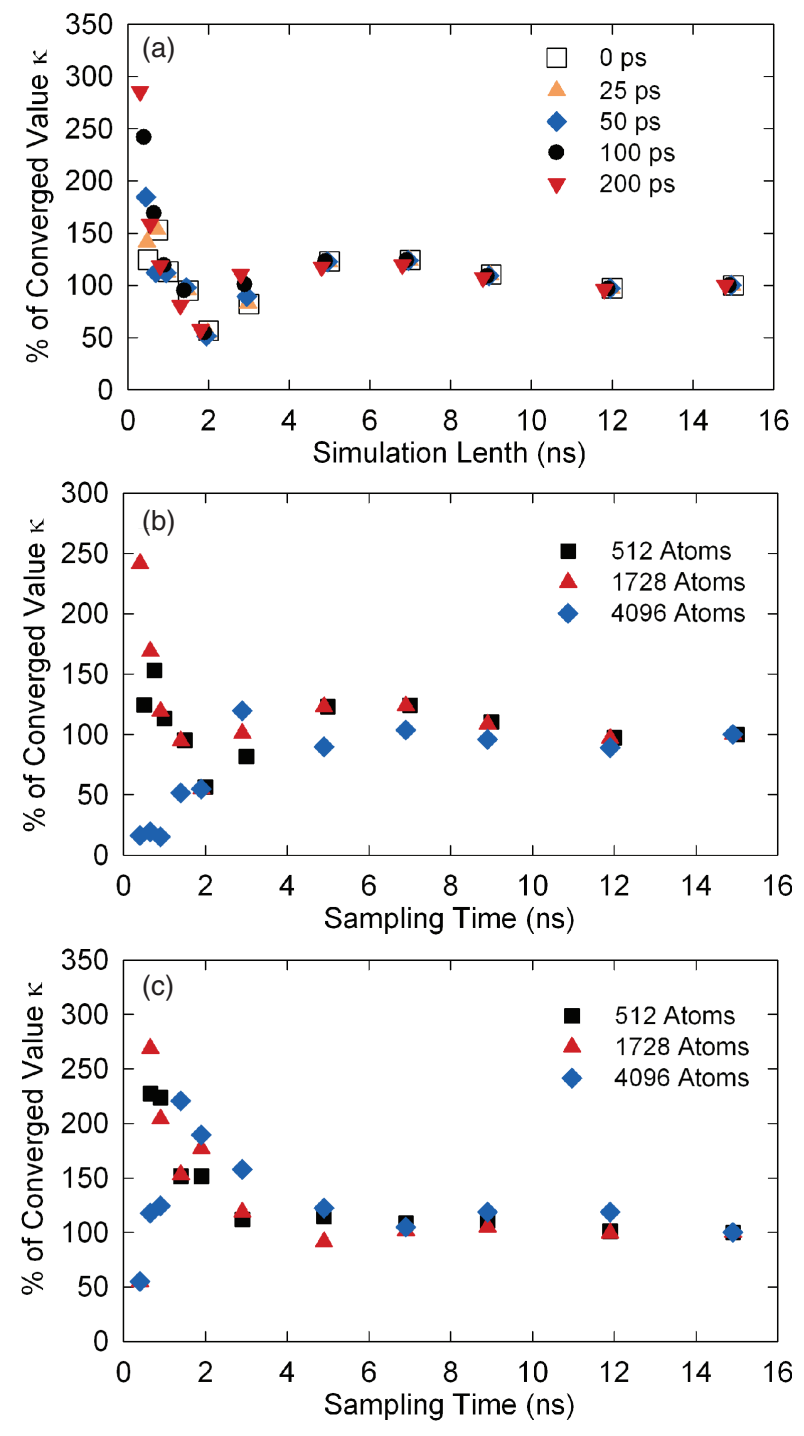

Fig. 2. Convergence of simulation results based on Green-Kubo formulation. (a) Percentage of the converged thermal conductivity of a 512 atom $(4 \times 4 \times 4$ unit cells $)$ system with different amounts of equilibration time and total simulation time. (b) Percentage of converged thermal conductivity value at $300 \mathrm{~K}$ for three systems of different size. (c) Percentage of converged thermal conductivity value at $600 \mathrm{~K}$ for three systems of different size.

The system sizes, for the Green-Kubo and relaxation time calculations, ranged between 320 and 4096 atoms. In each case, five independent simulations were conducted at each temperature in each direction to improve averaging. Once relaxation times were determined for modes in the symmetry directions, we used linear interpolation to calculate the relaxation times between data points. For the acoustic relaxation times corresponding to phonon frequencies below the lowest mode extracted, a $\tau \propto \nu^{-2}$ fit to the data set (as predicted by Klemens ${ }^{2}$ ) was used. With this scheme, a full spectrum of relaxation times for each polarization was generated and used to calculate the thermal conductivity and MFPs. 

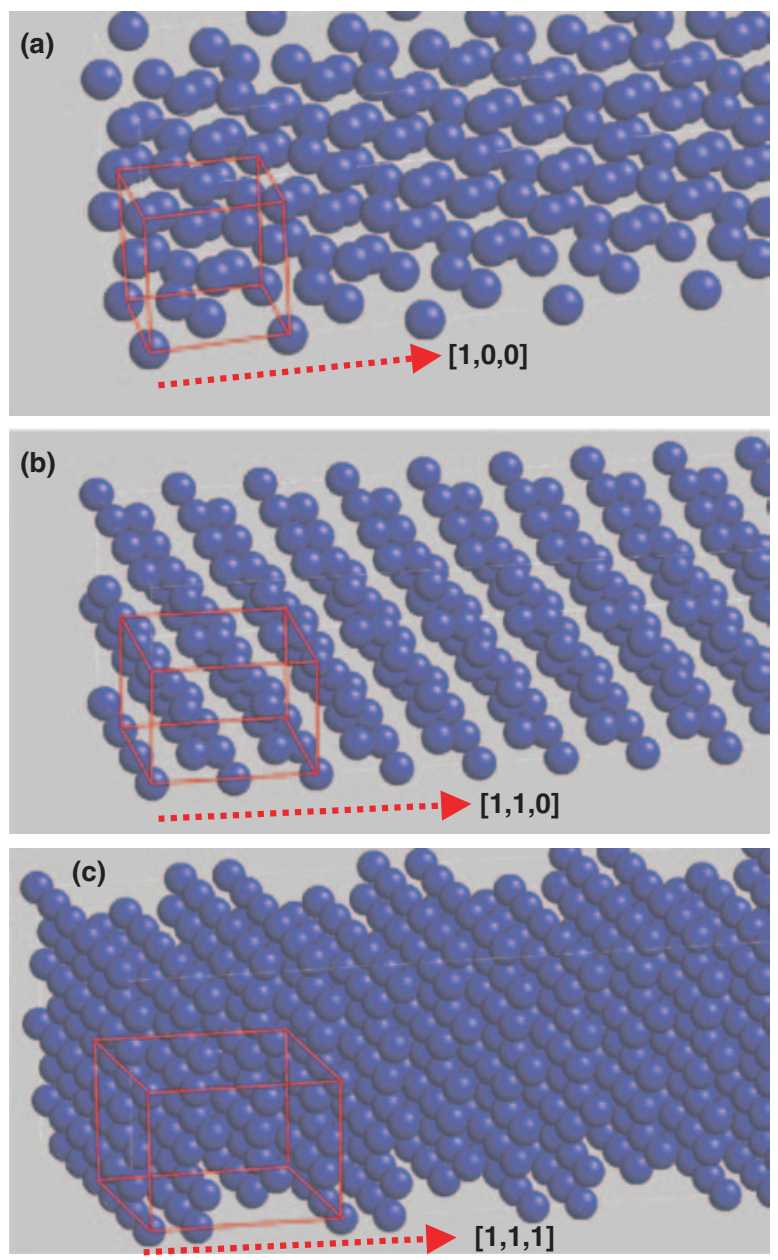

Fig. 3. Modified simulation cells. Modified unit cells indicated by red lines along different crystallographic directions, (a) $[1,0,0]$, (b) $[1,1,0]$, (c) $[1,1,1]$ (indicated by dotted arrows) aligned to the longest edge of the simulation domain.

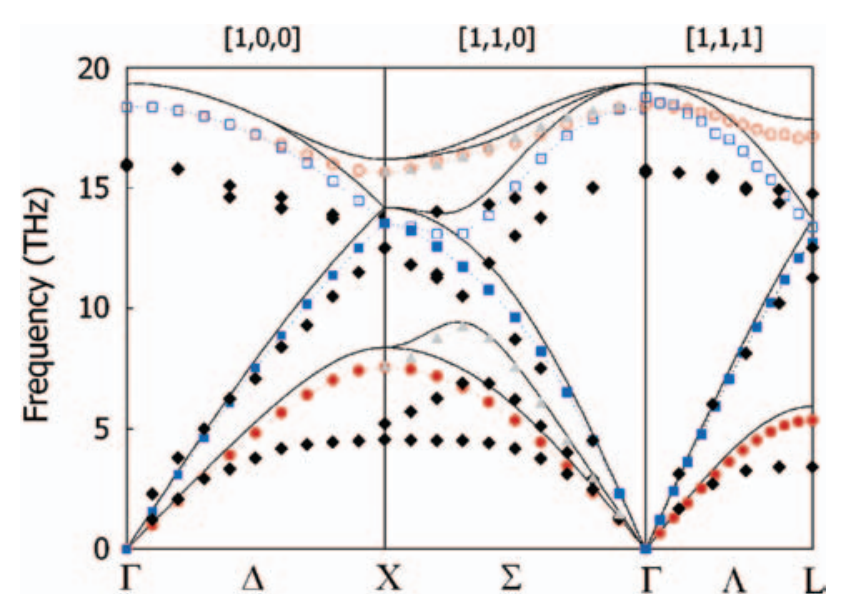

Fig. 4. Phonon dispersion. Phonon dispersion relations using frequencies extracted from MD simulations (symbols and dotted lines) and calculated with lattice dynamics (solid lines) as compared with experiments (diamonds). Reprinted with permission from [27], J. Hansen and I. McDonald, Theory of Simple Liquids, 2nd edn. (1986). (C) 1986, Academic Press, London.

\section{RESULTS AND DISCUSSION}

The frequencies extracted from the MD simulations and calculated from lattice dynamics are shown in Figure 4 with experimental values. ${ }^{30}$ The calculated dispersion matches the trends and magnitudes observed in experiments, but shows that the potential is overly stiff, which is a noted issue with many silicon potentials. ${ }^{29}$ To further test the accuracy of the potential, we conducted Green-Kubo simulations with 512 and 1728 atoms at ten temperatures. The results shown in Figure 5(a) capture the magnitude and trends observed in experiments ${ }^{31}$ suggesting that EDIP is adequately suited for studying the thermal conductivity of bulk silicon. The thermal conductivities calculated from Eq. (13) are shown in Figure 5(b). These results use the density of states summed over the Brillouin zone in combination with the group velocities and relaxation times extracted from the symmetry directions. When averaged, the results from each direction vary between $15-30 \%$. This acts as an estimate of the error associated with the assumption of isotropic phonon properties, which was employed in deriving Eq. (13).

The panels of Figures 6 through 9 show our results for the relaxation times at the ten temperatures considered, for each polarization in each of the symmetry directions. Each figure shows that the relaxation times are not generally monotonically decreasing with increasing frequency. Ladd et al. ${ }^{22}$ as well as McGaughey and Kaviany ${ }^{23}$ also observed similar non-monotonic behavior in their relaxation times for solid argon. The acoustic relaxation times show very strong frequency dependence, that approaches Klemen's ${ }^{2}$ prediction of $\tau \propto \nu^{-2}$ at the lower frequencies and higher temperatures. The more dispersive phonons with higher frequencies, however, exhibit other nonlinear characteristics that tend to relax as the temperature is

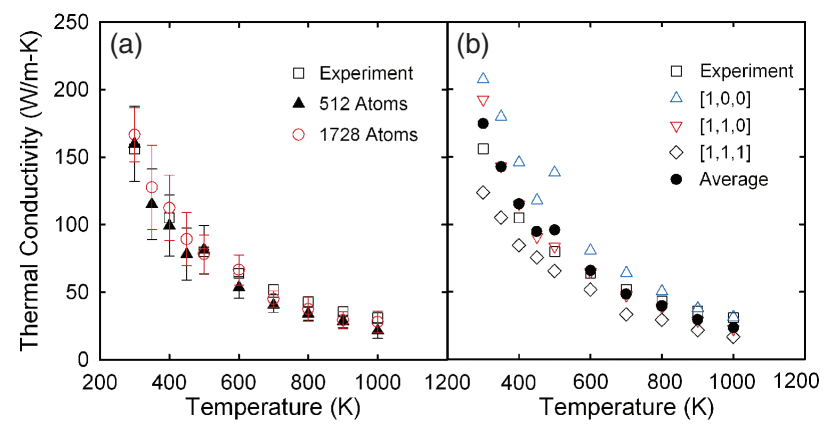

Fig. 5. Thermal conductivity of bulk silicon. (a) Green-Kubo thermal conductivity of a 512 atom $(4 \times 4 \times 4$ unit cell) and 1728 atom $(6 \times 6 \times 6$ unit cell system) compared with experiments. (b) Thermal conductivity using the BTE approach compared with experiments. Reprinted with permission from [28], R. Hardy, Phys. Rev. 132, 168 (1963). (C) 1963. The plot shows results generated from the density of states, averaged over the Brillouin zone, in combination with the phonon properties (group velocities and relaxation times) extracted along each symmetry direction. The average of the three directions is shown and the error bars indicate the error (standard deviation) associated with assuming isotropic phonon properties. 


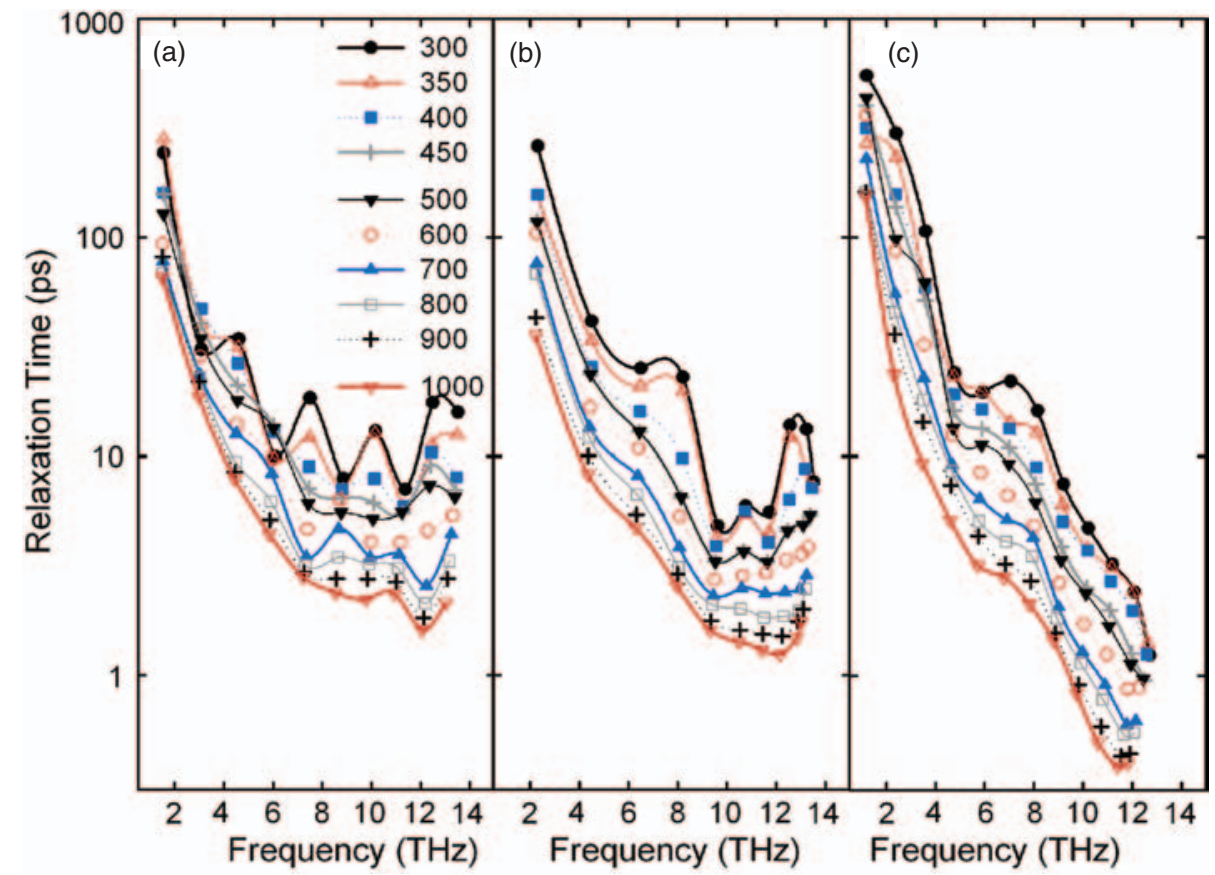

Fig. 6. Longitudinal acoustic (LA) phonon relaxation times. (a) Longitudinal acoustic phonon relaxation times extracted from normal mode decay times in the (a) $[1,0,0]$ (b) $[1,1,0]$ and (c) $[1,1,1]$ directions at ten different temperatures.

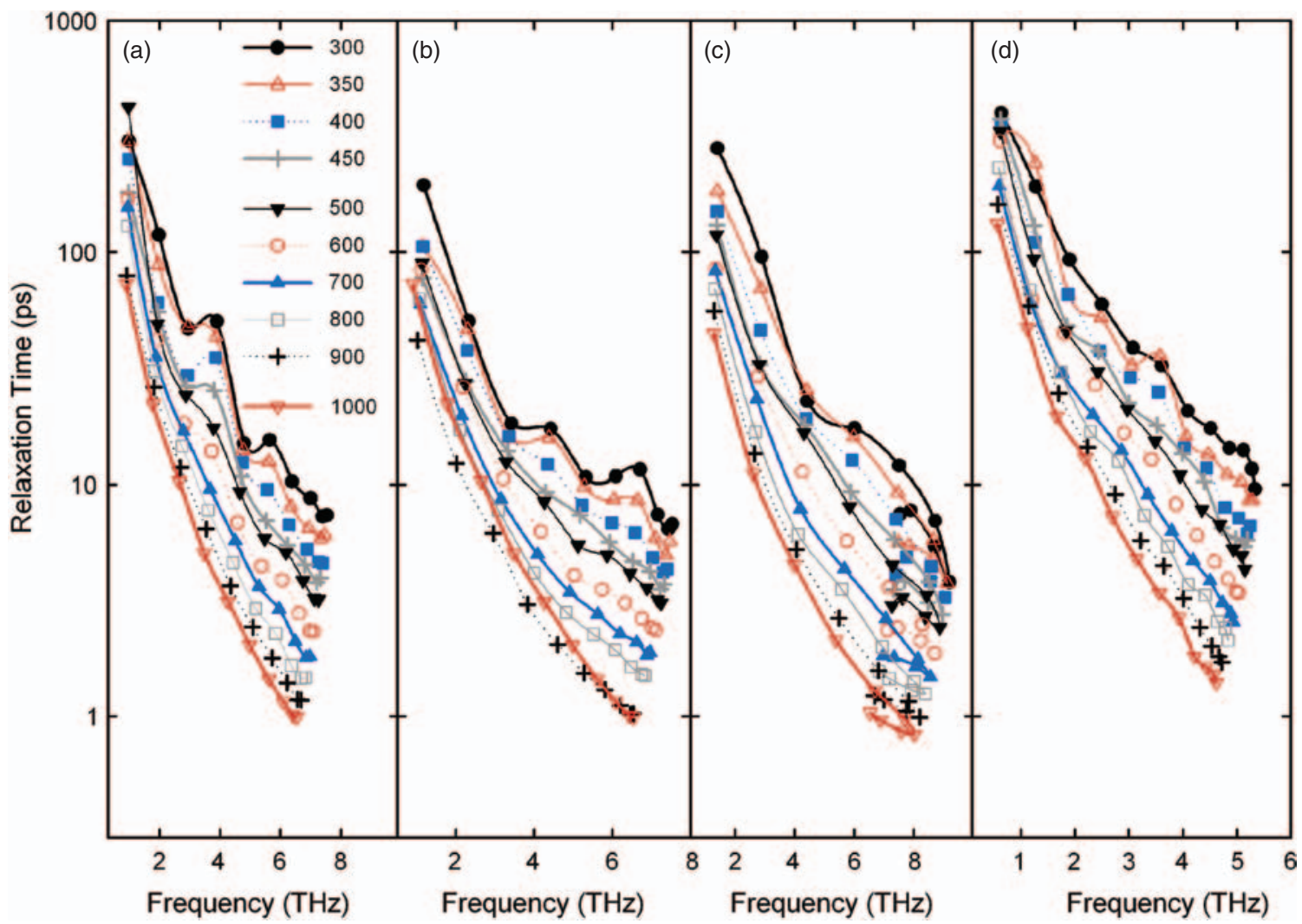

Fig. 7. Transverse acoustic (TA) phonon relaxation times. (a) Transverse acoustic phonon relaxation times extracted from normal mode decay times in the (a) $[1,0,0]$ (b) $[1,1,0]$ (c) $[1,1,0]$ and (d) $[1,1,1]$ directions at ten different temperatures. The transverse modes in the $[1,1,0]$ direction are non-degenerate. (c) Corresponds to the second TA branch of the dispersion, where the frequencies are not monotonically increasing. 


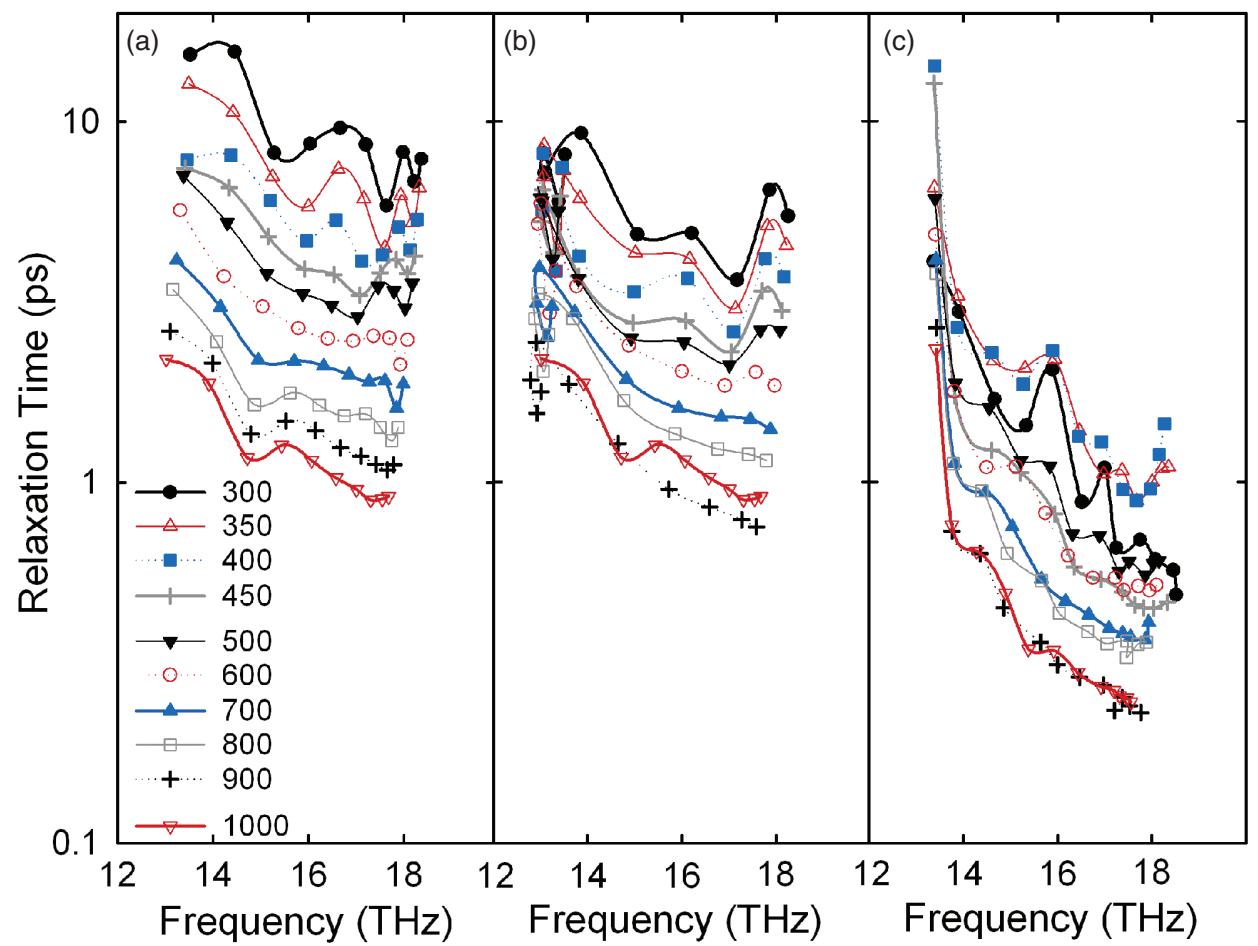

Fig. 8. Longitudinal optical (LO) phonon relaxation times. (a) Longitudinal optical phonon relaxation times extracted from normal mode decay times in the (a) $[1,0,0]$ (b) $[1,1,0]$ and (c) $[1,1,1]$ directions at ten different temperatures.

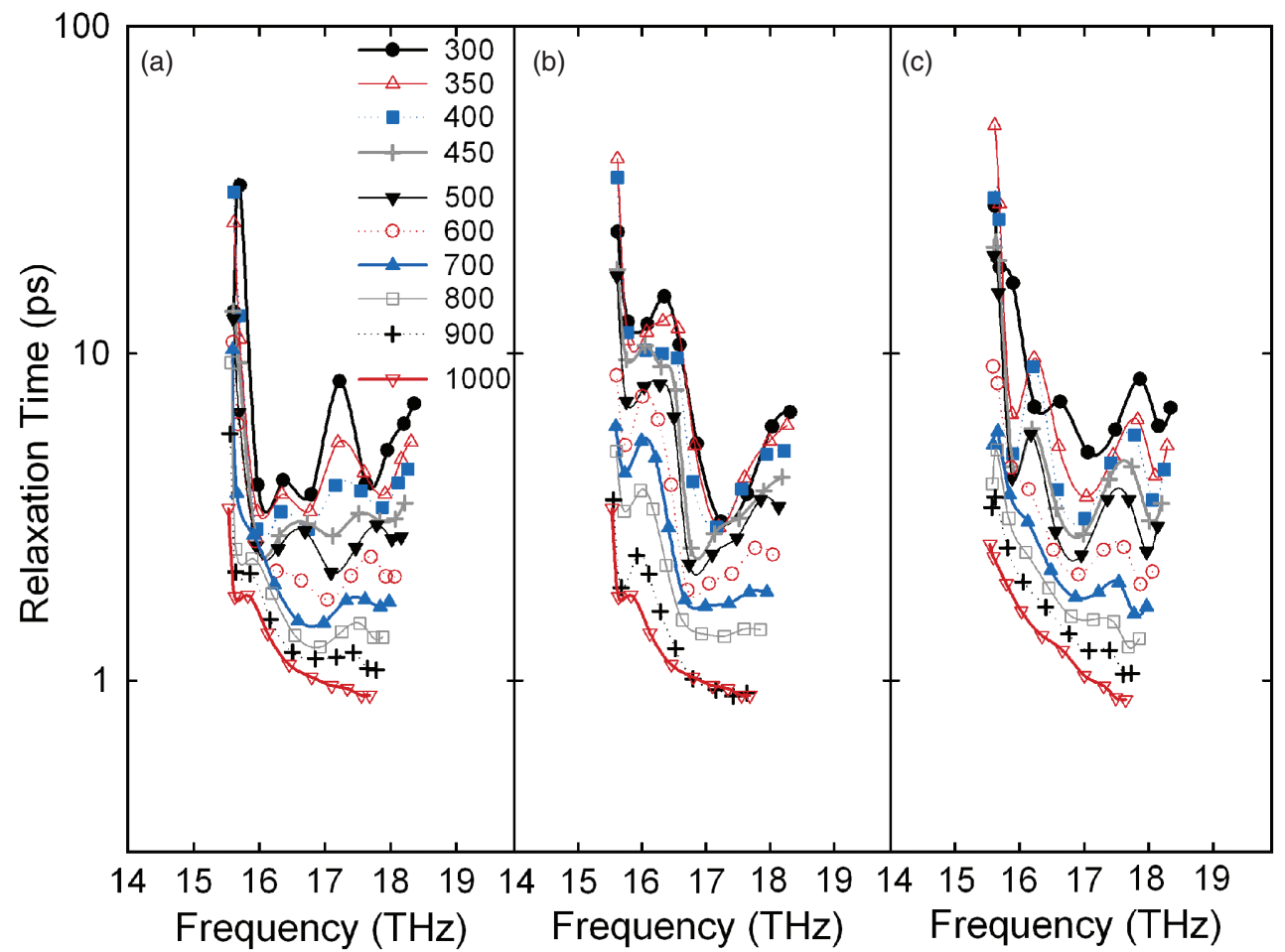

Fig. 9. Transverse acoustic (TO) phonon relaxation times. (a) Transverse optical phonon relaxation times extracted from normal mode decay times in the (a) $[1,0,0]$ (b) $[1,1,0]$ and (c) $[1,1,0]$ (non-degenerate) directions at ten different temperatures. 
increased. By fitting the acoustic relaxation times with a $\tau=A \cdot \nu^{-2} \cdot T^{-b}$ model we find $A=5.32 \cdot 10^{18}\left(\mathrm{~K}^{1.49} / \mathrm{sec}\right)$, $b=1.49$ for LA phonons and $A=5.07 \cdot 10^{18}\left(\mathrm{~K}^{1.65} / \mathrm{sec}\right)$, $b=1.65$ for TA phonons. Some of the previous analytical approaches ${ }^{2-4,20}$ to relaxation times have not predicted the stronger temperature dependence of thermal conductivity at higher temperatures. Srivasatava ${ }^{20}$ attributed this to temperature dependent anharmonicity, nonlinear dispersion and possibly four-phonon interactions. Calculating relaxation times with MD simulations includes all of these effects and captures the stronger temperature dependence. Omini and Sparavigna considered the effect of temperature dependent anharmonicity and showed stronger temperature dependence closer to what is observed in experiments, suggesting that any remaining discrepancy may be due to higher order interactions, which are included in our calculation. Srivastava also considered optical phonons in his work, ${ }^{20}$ using the Einstein approximation (constant optical frequency), and showed they significantly reduce thermal conductivity by scattering acoustic phonons, but do not alter the temperature dependence. Our results for optical phonon relaxation times exhibited weak frequency dependence (approximately constant) and were about an order of magnitude smaller than the acoustic relaxation times. The temperature dependence is similar to the trends observed for acoustic relaxation times, which agrees with Srivastava's calculations. The transverse optical phonon relaxation times for the $[1,1,1]$ direction have been omitted because they were lower than other optical relaxation times by an order of magnitude and exhibited very inconsistent behavior.

Our frequency and polarization dependent methodology allowed for direct evaluation of the contributions from different branches of phonon states. Our results indicate that LA phonons contribute roughly $45 \%$ to the thermal conductivity, which is in excellent agreement with Omini and Sparavigna ${ }^{21}$ and also qualitatively agrees with Ju and Goodson's deduction from experimental observations. ${ }^{16}$ The results indicate that on average the two TA polarizations contribute roughly 30\% (TA-1) and 20\% (TA2) individually, while the optical polarizations contribute the remainder. The strong frequency dependence of the acoustic relaxation times implies that effective or frequency averaged values for the MFPs can inaccurately represent the characteristics of phonon transport. To examine the idea further we recalculated the thermal conductivity using different combinations of frequency averaged phonon transport properties. As a qualitative measure of the error introduced by neglecting the spectral dependence of each component to the thermal conductivity (specific heat, group velocity and relaxation time), we compared the thermal conductivity with different combinations of effective values to the BTE-average thermal conductivity (in Fig. 5(b)), where each component's frequency dependence was included. To define an effective or frequency averaged property $\bar{\eta}_{p}$ we integrated over the frequency spectrum, using the density of states as a weighting function, to generate an arithmetic average over phonon states (effective value) for each polarization

$$
\bar{\eta}_{p}=\frac{\int_{0}^{\nu_{\max }} \eta_{p}(\nu) \cdot D_{p}(\nu) \cdot d \nu}{\int_{0}^{\nu_{\max }} D_{p}(\nu) \cdot d \nu}
$$

where $\eta_{p}=h \nu \cdot\left(d f_{0} / d T\right)$ for the specific heat, $\eta_{p}=v_{p}(\nu)$ for the group velocities $\eta_{p}=\tau_{p}(\nu)$ for the relaxation times and the subscript $p$ implies that we have retained the polarization dependence. This effective value, a constant for each polarization, was then used to calculate the thermal conductivity with the full frequency dependence considered for the remaining properties inside the integral of Eq. (13). This provided a qualitative measure of the relative error associated with effective or frequency averaged treatment of a particular phonon property. Figure 10 shows the percentage difference of the thermal conductivities from the BTE-averaged values when effective properties are used. The legend lists the properties that retained their frequency dependence in the integral (Eq. (13)). Figure 10 shows that preserving the frequency dependence of the group velocities and relaxation times together leads to the least amount of error. Treating other combinations of properties as frequency independent, leads to $\sim 20 \%-40 \%$ error, except when all three properties are treated as frequency independent, in which case the thermal conductivity is over predicted by $\sim 95 \%$. The results in Figure 10 generally show that considering the frequency dependence of the phonon properties is highly important as we qualitatively show that the use of frequency averaged properties can lead to slightly larger errors than the assumption of isotropic phonon properties.

To understand the strength of size effects we used isotropic and polarization averaged phonon MFPs, which span seven orders of magnitude over three orders of magnitude in frequency. This large spread of phonon MFPs is due to the diverging $\tau \propto \nu^{-2}$ dependence at low frequencies and phonons with group velocities that approach

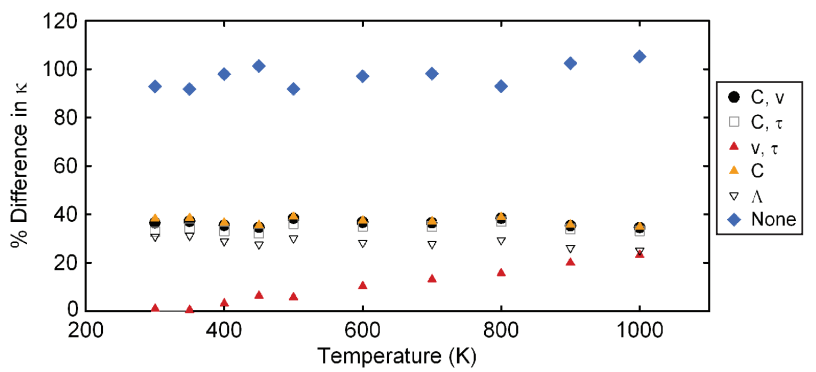

Fig. 10. Deviation in thermal conductivity from frequency averaged properties. Percentage change in thermal conductivity when compared to the spectral dependent (directionally averaged) BTE values. The figure legend lists the variables that remained inside the frequency dependent integral. The remaining variables, not listed in the legend, were replaced by a constant equal to the average over phonon states as described in Eq. (20). 
zero near the Brillouin zone boundary. In Figure 11(a) we show (in arbitrary units) the number of phonon states corresponding to the range of MFPs, noting that at room temperature most phonons have a MFP between $10 \mathrm{~nm}$ and 1 micron. In Figure 11(b) we show the thermal conductivity and its accumulation with respect to the average phonon MFPs. ${ }^{9}$

$$
\frac{1}{\kappa} \cdot \sum_{p} \frac{1}{3} \int_{0}^{\nu^{\prime}} C \cdot v \cdot \Lambda\left(\nu^{\prime}\right) \cdot d v
$$

This plot shows that the relatively few states that have MFPs much longer than 1 micron contribute $\sim 35 \%$ to the thermal conductivity at room temperature. This contrasts the commonly held notion that thermally important phonons only have MFPs on the order of micron at cryogenic temperatures. Our results show that only a small number of phonon states carry energy beyond 1 micron, yet they are large contributors to the thermal conductivity and are thus impacted by boundaries at the micrometer length scale. Figure 11(b) shows that at room temperature phonons with MFPs between $100 \mathrm{~nm}$ and 10 micron, which represent a minority of the phonon states, comprise $\sim 70 \%$ of the thermal conductivity at room temperature. Figure 12 shows the contributions to thermal conductivity with respect to polarization averaged wavelengths. This figure shows that $80 \%$ of the thermal conductivity is attributed to phonons with wavelengths less than $10 \mathrm{~nm}$.
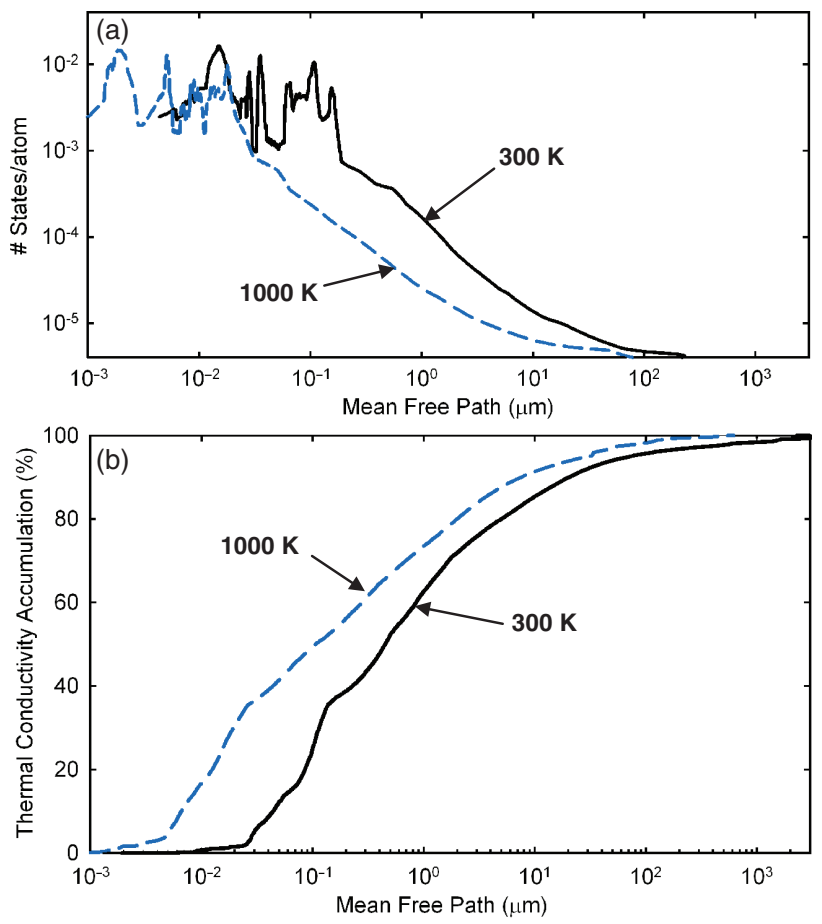

Fig. 11. Density of states and thermal conductivity accumulation with respect to MFPs. (a) The density of phonon states (arbitrary units) with respect to the average phonon MFPs at $300 \mathrm{~K}$ (solid line) and $1000 \mathrm{~K}$ (dashed line). (b) Percentage of thermal conductivity accumulation at $300 \mathrm{~K}$ (solid line) and $1000 \mathrm{~K}$ (dashed line).

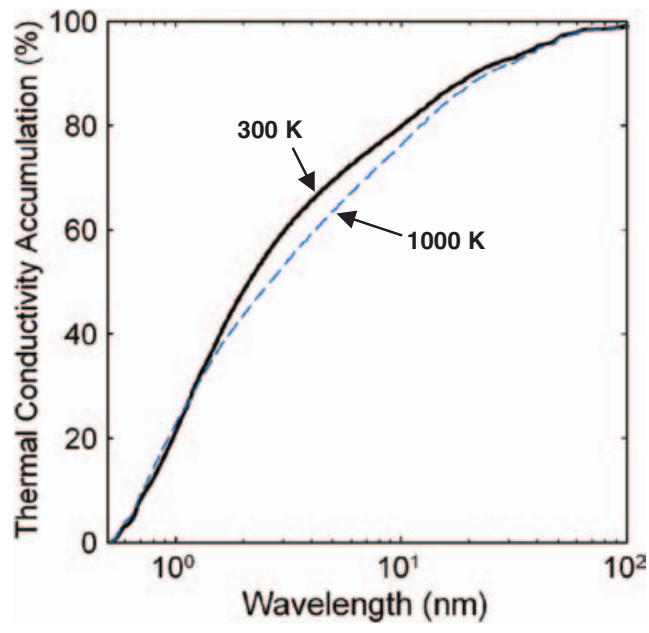

Fig. 12. Thermal conductivity accumulation with respect to wavelength. Percentage of thermal conductivity accumulation at $300 \mathrm{~K}$ (solid line) and $1000 \mathrm{~K}$ (dashed line).

These results provide new explanation of the strong size effects observed for silicon microstructures. ${ }^{17,18}$ Our results in Figures 11 and 12 also indicate that even though the MFPs are much shorter at $1000 \mathrm{~K}$, the spectral dependence is not a strong function of temperature and the same phonons are responsible for the energy transport.

\section{CONCLUSION}

A major obstacle to analytical study of phonon-phonon scattering has been the relative scaling of the contributions from different polarizations. Although previous works have generated semi-empirical expressions for relaxation times, the fitting parameters could not be determined reliably and questions have continued to linger. As an alternative we have presented a numerical simulation technique that reliably provides the details of phonon transport properties and can be applied to any crystalline solid. In our various simulations of bulk silicon we were able to extract relaxation times for each polarization in different directions and investigated the spectral dependence of silicon's thermal conductivity. Our results indicate that the contribution from longitudinal acoustic phonons is comparable to that of the two transverse acoustic branches. Our study of the spectral dependence showed that effective or frequency averaged phonon transport properties can lead to significant errors $\sim 20-40 \%$, while anisotropy causes properties to vary by $15-30 \%$. Our MFP results indicate also that relatively few phonons with MFPs greater than 1 micron contribute $35 \%$ to the thermal conductivity. Such strong contributions from these phonons provide explanation for the size effects observed in various silicon microstructures. ${ }^{17,18}$ The relaxation time results also provide useful insight for understanding size effects in silicon while providing more reliable input for other modeling and simulation techniques to study micro/nanoscale heat transfer. 
Acknowledgments: We acknowledge financial support from NASA, DOE and the DOE computational science graduate fellowship to A.H. We also acknowledge computational resources provided by Intel Corporation and discussion with S. Volz.

\section{References}

1. R. Peierls, Ann. Physik 3, 1055 (1929).

2. P. Klemens, Proc. Roy. Soc. 108, A208 (1951).

3. J. Callaway, Phys. Rev. 113, 1046 (1959).

4. M. Holland, Phys. Rev. 132, 2461 (1963).

5. P. Klemens, Solid-State Physics 7, 1 (1958).

6. P. Carruthers, Rev. Mod. Phys. 33, 92 (1961).

7. M. Holland, Phys. Rev. 134, A471 (1964).

8. G. P. Srivastava, The Physics of Phonons, Adam Hilger, New York (1990).

9. C. Dames, B. Poudel, W. Z. Wang, J. Huang, F. Ren, Y. Sun, J. Oh, C. Opeil, M. Naughton, and G. Chen, Appl. Phys. Lett. 87, 031901 (2005)

10. D. Li, Y. Wu, P. Kim, L. Shi, P. Yang, and A. Majumdar, Appl. Phys. Lett. 83, 2934 (2003).

11. R. Yang and G. Chen, Phys. Rev. B 69, 195216 (2004).

12. E. Pop and K. Goodson, J. Electronic Packaging 128, 102 (2004).
13. T. Harman, P. J. Taylor, M. P. Walsh, and B. E. LaForge, Science 297, 2229 (2002).

14. C. Duck-Young, T. Hogan, P. Brazis, M. Rocci-Lane, C. Kannewurf, M. Bastea, C. Uher, and M. Kanatzidis, Science 287, 1024 (2000).

15. G. Chen, Phys. Rev. B 57, 14958 (1998).

16. Y. Ju and K. Goodson, App. Phys. Lett. 74, 3005 (1999).

17. D. Song and G. Chen, Appl. Phys. Lett. 84, 687 (2004).

18. N. Savvides and H. J. Goldsmid, J. Phys. C 6, 1701 (1973).

19. R. Hamilton and J. Parrot, Phys. Rev. 178, 1284 (1969).

20. G. P. Srivastava, J. Phys. Chem. Solids 41, 357 (1980).

21. M. Omini and A. Sparavigna, Nuovo Cimento 19, 1537 (1997).

22. A. Ladd, B. Moran, and W. Hoover, Phys. Rev. B 34, 5058 (1986).

23. A. McGaughey and M. Kaviany, Phys. Rev. B 69, 094303 (2004).

24. S. Volz and G. Chen, Phys. Rev. B 61, 2651 (2000).

25. P. Schelling, S. Phillpot, and P. Keblinski, Phys. Rev. B 64, 144306 (2002).

26. L. Sun and J. Murthy, Appl. Phys. Lett. 89, 171919 (2006).

27. J. Hansen and I. McDonald, Theory of Simple Liquids, 2nd edn., Academic Press, London (1986).

28. R. Hardy, Phys. Rev. 132, 168 (1963).

29. M. Bazant and E. Kaxiras, Phys. Rev. B 56, 8542 (1997).

30. P. Zschack, P. Jemian, J. Tischler, H. Chen, and T. Chiang, Phys. Rev. Lett. 83, 3317 (1999).

31. C. J. Glassbrenner and G. Slack, Phys. Rev. 134, 4A 1058 (1963).

Received: 10 December 2006. Accepted: 24 April 2007. 